\title{
Adaptação e Validação da Escala de Perfeccionismo Almost Perfect Scale - Revised para o Português Brasileiro
}

\author{
Flávio Henrique dos Reis Soares ${ }^{1}$ (D) \\ Universidade Federal de Minas Gerais, Belo Horizonte-MG, Brasil \\ Anelisa Vaz de Carvalho (D) \\ Universidade do Estado de São Paulo, Ribeirão Preto-SP, Brasil \\ Eduardo Keegan (B) \\ Universidad de Buenos Aires, Argentina \\ Carmem Beatriz Neufeld (D) \\ Universidade do Estado de São Paulo, Ribeirão Preto-SP, Brasil \\ Marcela Mansur-Alves (1D) \\ Universidade Federal de Minas Gerais, Belo Horizonte-MG, Brasil
}

\section{RESUMO}

O perfeccionismo é um construto multidimensional cujo estudo vem aumentando desde os anos de 1990. Uma das escalas mais utilizadas para sua mensuração é a Almost Perfect Scale - Revised (APS-R), composta por três dimensões: Padrões, Discrepância e Ordem. Devido à ausência de medidas como essa no Brasil, este estudo objetivou adaptar e analisar as propriedades psicométricas da APS-R em uma amostra brasileira, por meio da análise de sua estrutura interna e levantamento de sua confiabilidade. Participaram 787 universitários (79,3\% do sexo feminino), idade média de 25,98 anos $(D P=7,29)$ que responderam à APS-R e a um questionário sociodemográfico. Os itens adaptados mostraram IVC adequados: equivalência semântica $(0,99)$, cultural $(0,94)$ e conceitual $(0,94)$. Dentre os modelos estruturais testados, o bifatorial mostrou-se superior aos demais $\left(\chi^{2}=452,371 ; \mathrm{TLI}=0,94 ; \mathrm{CFI}=0,96 ; \mathrm{RMSEA}=0,047\right)$. Assim, a escala apresentou propriedades psicométricas satisfatórias para uso no Brasil, tanto no que se refere à sua validade quanto confiabilidade. São discutidas limitações e implicações do estudo.

Palavras-chave: Almost Perfect Scale (APS-R); perfeccionismo; propriedades psicométricas; modelagem de equação estrutural exploratória.

\section{ABSTRACT - Adaptation and Validation for Brazilian Portuguese of the Revised Almost Perfect Scale}

The study of perfectionism as a multidimensional construct has increased since the 1990's. The Almost Perfect Scale (APS-R) is widely used across studies to measure perfectionism; it is composed by three dimensions: High Standards, Discrepancy, and Order. However, despite increasing interest in the measurement of perfectionism, there were no scales available for Brazilian-Portuguese. Accordingly, we aimed to adapt the APS-R and to analyze the psychometric properties and test confirmatory models for the Brazilian version of the Almost Perfect Scale (APS-R Brasil). A sample of university students $(n=787)$ with a mean age 25.98 years $(S D=7.29), 79.3 \%$ women, completed the APS-RB. The adapted items demonstrated semantic, cultural and conceptual adequacy (all CVI<90). The ESEM two factor model, showed the better fit index $\left(\chi^{2}=452.371 ;\right.$ TLI=0.94; CFI $\left.=0.96 ; \mathrm{RMSEA}=0.047\right)$. The Brazilian version of APS-R was shown to be valid and reliable. Limitations and implications of the study are discussed. Keywords: Almost Perfect Scale (APS-R); perfectionism; psychometric properties; exploratory structural equation modeling.

\section{RESUMEN - Adaptación y Validación de la Escala de Perfeccionismo Almost Perfect Scale - Revised para el Portugués} Brasileño

El estudio del perfeccionismo como un constructo multidimensional ha aumentado desde los años 90. La escala Almost Perfect Scale-Revised (APS-R), que se usa ampliamente en los estudios del perfeccionismo, está compuesta por tres factores: altos estándares, la percepción de la discrepancia y el orden. Sin embargo, a pesar del creciente interés en la medición del perfeccionismo, todavía no hay escalas disponibles para el portugués brasileño. Por lo tanto, el objetivo de esta investigación fue adaptar y analizar las propiedades psicométricas, y probar modelos confirmatorios para la APS-R en una muestra brasileña. Dicha muestra se compuso por estudiantes universitarios $(n=787)$ con una edad media de 25,98 años $(D E=7,29)$ y $79,3 \%$ mujeres. Los ítems adaptados demostraron adecuación semántica, cultural y conceptual (todos los IVC <90). El modelo ESEM Bifactor mostró el mejor índice de ajuste $\left(\chi^{2}=452,371 ; \mathrm{TLI}=0,94 ; \mathrm{CFI}=0,96 ; \mathrm{RMSEA}=0,047\right)$. La versión brasileña de APS-R demostró ser válida y fiable. Aparte, se discute las limitaciones e implicaciones del estudio.

Palabras clave: Almost Perfect Scale (APS-R); perfeccionismo; propriedades psicométricas; modelos de ecuaciones estructurales exploratorios. 
O perfeccionismo é concebido como uma característica multidimensional da personalidade composta por aspectos associados a desfechos negativos e positivos (Stairs, Smith, Zapolski, Combs, \& Settles, 2012; Stoeber et al., 2006). Pode-se definir o perfeccionismo como a tendência pessoal à definição e busca de padrões de desempenho extremamente elevados ligados a um excesso de autocriticismo (Frost, Marten, Lahart, \& Rosenblate, 1990; Stoeber, 2018). Nas últimas décadas, os estudos em conceituação e avaliação do perfeccionismo aumentaram exponencialmente (Flett \& Hewitt, 2016). A avaliação do perfeccionismo tem sido cada dia mais relevante para compreensão de diferentes desfechos relacionados à saúde mental, tais como: transtornos ansiosos, depressão, transtornos alimentares, transtorno de personalidade obsessivo-compulsiva, transtorno obsessivo compulsivo, além do perfeccionismo ser considerado como fator responsável por pior resposta a tratamentos psicoterápicos (Egan, Wade, Shafran, \& Antony, 2014; Newby et al., 2017; Pinto et al., 2017; Sherry, Richards, Sherry, \& Stewart, 2014; Smith, Saklofske, Yan, \& Sherry, 2017).

Os avanços na avaliação do perfeccionismo devem-se, em grande parte, à criação de escalas fidedignas e válidas. Dentre os modelos utilizados para embasar a construção de medidas de perfeccionismo, o modelo trifatorial proposto por Slaney, Rice, Mobley, Trippi, e Ashby (2001) advoga pela existência de componentes positivos e negativos dessa característica. Esse modelo é composto por três dimensões básicas: Padrões $(\mathrm{P})$, Discrepância (D) e Ordem (O). Segundo Slaney et al. (2001, 1996), a dimensão de Padrões pessoais se refere à tendência pessoal ao estabelecimento de padrões de desempenho muito elevados. A dimensão de Discrepância trata do julgamento individual e crítica excessiva com relação ao desempenho pessoal e da percepção de estar aquém do desempenho considerado ideal. A dimensão de Ordem intenciona operacionalizar a tendência à organização e ao asseio. A combinação entre essas dimensões aponta que, enquanto um alto nível de Padrões e Ordem é considerado positivo ou inócuo, um nível elevado de discrepância é considerado prejudicial e está ligado a fatores e tendências desadaptativas da personalidade (Chan, 2010; Rice, Ashby, \& Slaney, 2007). Em proposta mais recente, Stoeber et al. (2006) denominaram o aspecto adaptativo (positivo) do perfeccionismo como Esforço Perfeccionista (perfectionist strivings) e o aspecto desadaptativo (negativo) como Preocupações Perfeccionistas (perfectionist concerns) em uma tentativa de organizar as diferentes dimensões do perfeccionismo propostas em diferentes modelos teóricos. No modelo de Slaney et al. (2001), a dimensão discrepância carregaria em preocupações perfeccionistas, enquanto a dimensão Padrões carregaria em esforços perfeccionistas. A dimensão de ordem tem sido considerada elemento não essencial para compreensão do construto, ou seja, defende-se de que nem todos os perfeccionistas poderiam ser caracterizados por tendência à organização e ao asseio (Frost et al., 1990; Mobley, Slaney, \& Rice, 2005; Stoeber et al., 2006).

Em 1996, foi construída a primeira versão da escala de perfeccionismo, Almost-Perfect Scale. A proposta da escala foi impulsionada pela necessidade de avaliação tanto dos aspectos positivos como dos aspectos negativos do perfeccionismo, principalmente no que diz respeito às suas implicações clínicas. Posteriormente, essa escala passou por um refinamento culminando na Almost Perfect Scale Revised (APS-R) como é reconhecida e utilizada hoje. Em sua versão mais atual, a escala apresenta validade estrutural e consistência interna adequada em diversas culturas (Mobley et al., 2005). Sua versão revisada norte-americana, composta por 23 itens apresentou índices adequados de confiabilidade para as dimensões de Discrepância, Padrões e Ordem, com $\alpha$ de Cronbach de 0,91, 0,85 e 0,82 , respectivamente, e ajuste estrutural satisfatório, como indicado pelos índices de ajustes da análise fatorial confirmatória, $\chi^{2}=459,22$; SRMR $=0,08, \mathrm{RMSEA}=0,07$ e CFI $=0,90$. Contudo, há variações quanto a esse ajuste do modelo teórico aos dados empíricos em amostras não norte-americanas. Por exemplo, Cazan (2016), por meio de análise fatorial confirmatória (AFC), conseguiu melhor ajuste em um modelo de dois fatores. Denominou o primeiro, composto pela dimensão de Discrepância, de Desadaptativo $(\alpha=0,91)$ e o segundo, composto pelas dimensões de Padrão e Ordem, de Adaptativo $(\alpha=0,88)$. Embora difira do modelo original, essa alternativa vai ao encontro da proposta de Stoeber et al. (2006) relacionado aos esforços perfeccionistas (Padrões e Ordem) e preocupações perfeccionistas (Discrepância). Por sua vez, na Espanha, Sastre-Riba, Pérez-Albéniz e Fonseca-Pedrero (2016) também examinaram as propriedades psicométricas da APS-R. A estrutura derivada da análise fatorial exploratória indicou a retenção de três fatores e, dessa vez, a AFC apresentou índices de ajuste adequados para esse modelo. A estrutura de três dimensões também foi replicada em culturas não ocidentais. Tanto entre estudantes indianos, quanto entre estudantes chineses e coreanos, o modelo de três fatores foi replicado com consistência interna da escala variando de 0,78 a 0,92 (Chan, 2010; Park, 2009; Wang, Puri, Slaney, Methikalam, \& Chadha, 2012). Apesar das diversas adaptações culturais da APS-R, na América Latina, há apenas um estudo psicométrico da escala, adaptada e traduzida para falantes do espanhol na Argentina (Arana, Keegan, \& Rutsztein, 2009). Nesse contexto, foram extraídos, por meio de análise fatorial exploratória (AFE), os três fatores conforme propostos para APS-R. Contudo, o estudo careceu da testagem de um modelo confirmatório, deixando dúvidas sobre o melhor modelo teórico para descrição dos perfeccionistas em amostras latino-americanas. Assim, acerca da generalidade de um modelo de perfeccionismo baseado na APS-R, poder-se-ia argumentar que os estudos atuais se corroboram apenas em parte devido à variação das estruturas replicadas, ora de dois, ora de três fatores. 
Quanto ao Brasil, não existem dados sobre a estrutura do perfeccionismo para essa população que, embora próxima geograficamente, difere drasticamente dos indivíduos argentinos, tanto no que se refere a fatores culturais quanto idiomáticos. Nesse sentido, o presente estudo pretendeu traduzir, adaptar culturalmente e levantar as propriedades psicométricas da APS-R para uma amostra de estudantes universitários brasileiros (adultos emergentes) por meio da análise da confiabilidade e estrutura interna do instrumento. Em caráter secundário, este estudo teve por objetivo testar uma estrutura bifatorial para o instrumento, considerando que existem propostas para a criação de um escore geral de perfeccionismo para a APS-R e outras escalas avaliando o construto, com consequente utilização desse escore como indicador para uso clínico, além dos escores nos componentes específicos (cf., Smith, Saklofske, Yan, \& Sherry, 2017; Frost et al., 1990; Slaney et al. 2001). Não obstante esse interesse recente, há poucos estudos visando sustentar, empírica e teoricamente, a utilização desse escore geral para perfeccionismo (Smith, \& Saklofske, 2017; Gäde, Schermelleh-Engel, \& Klein, 2017).

No presente estudo, foi hipotetizado que a versão adaptada seria válida em seu conteúdo conceitual e semântico e que o perfeccionismo seria adequadamente descrito também por um modelo bifatorial. Dessa maneira, permitiu-se que os itens carregassem livremente no fator latente proposto e fixou-se em 0 a carga nos demais fatores da APS-R, deixando a estimativa livre. Considerando as dimensões apresentadas anteriormente, esperava-se que os índices de ajuste do modelo bifatorial fossem uma alternativa para APS-R Brasil. Para a versão da escala em português brasileiro a ser testada, este trabalho baseou-se nas diretrizes gerais para adaptação e validação de instrumentos disponibilizadas pela International Test Comission (2016).

\section{Métodos}

O método do presente estudo está dividido em duas etapas. Na primeira etapa, serão apresentados os participantes e procedimentos do estudo de tradução e adaptação transcultural em suas fases e, na segunda etapa, os procedimentos envolvidos no estudo de validação. $\mathrm{O}$ projeto foi aprovado pelo Comitê de Ética em Pesquisa da Universidade Federal de Minas Gerais (CAAE 63461916.4.0000.5149).

\section{Estudo 1 - Processo de Tradução e Adaptação Cultural da Escala}

Após autorização dos autores da escala original para tradução e adaptação, esta passou pelas etapas indicadas pela International Test Comission (2016, (a) tradução do instrumento original (forward translation); (b) tradução reversa (backtranslation); (c) apreciação formal de equivalência; e (d) avaliação final por especialistas na área (julgamento de juízes quanto à equivalência da síntese dos itens obtidos nas etapas anteriores).

\section{Participantes}

Foram convidadas 12 profissionais para a etapa de adaptação do instrumento. $\mathrm{Na}$ fase de tradução, participaram cinco tradutores com proficiência em inglês e experiência na área de Psicologia. Na fase de tradução-reversa, participaram dois tradutores falantes nativos do inglês e com proficiência no português brasileiro. $\mathrm{Na}$ etapa de revisão do conteúdo dos itens, participaram cinco juízes, todos eles com experiência em Instrumentação Psicológica, Terapia Cognitivo-Comportamental ou ambas as áreas.

\section{Materiais/Instrumentos}

Formulário da avaliação de juízes: esse formulário continha todos os 23 itens da Almost Perfect Scale traduzidos e adaptados e foi utilizado na quarta fase do processo de adaptação do instrumento para avaliar cada um dos itens quanto: a clareza de linguagem (a linguagem utilizada é clara para os respondentes?), pertinência básica (os itens representam o construto?), relevância teórica (avaliação do grau de relação entre os itens e a teoria) e dimensão teórica (os itens representam cada uma das dimensões da APS-R?). Para cada um dos itens, as respostas eram dadas por meio de uma escala do tipo Likert de 4 pontos: 1 -Inadequado, 2 - Pouco Adequado, 3 - Adequado e 4 - Muito Adequado. Após registrar a avaliação dos itens, os juízes deveriam assinalar, no questionário a qual dimensão os itens remetiam. As definições de cada dimensão estavam destacadas nas instruções do formulário. Por fim, havia um espaço reservado para sugestões e comentários relacionados a alteração dos itens.

\section{Procedimentos de Coleta dos Dados}

Os procedimentos para obtenção da versão adaptada da APS-R seguiram as diretrizes da International Test Comission (2016). Assim, na primeira fase cinco profissionais com alta proficiência na língua inglesa traduziram o instrumento original para o português brasileiro, dando origem à cinco versões traduzidas. Em seguida, todas as versões foram comparadas e criou-se uma síntese levando em consideração as peculiaridades da língua brasileira e a preservação do sentido dos itens. Na segunda fase, a versão-síntese da APS-R Brasil foi retrotraduzida por dois falantes nativos da língua inglesa com proficiência em português. Na terceira fase, a versão retrotraduzida foi avaliada por um professor especialista em psicometria a fim de atestar a preservação do sentido dos itens. Por fim, a versão brasileira foi enviada para análise de conteúdo de juízes, etapa na qual foi verificada a adequação semântica e conceitual da escala. A banca de avaliação foi composta por dois juízes com comprovada expertise em 
Terapia Cognitivo Comportamental (TCC) e um juiz com Expertise em Psicometria além de TCC.

\section{Procedimentos de Análise de Dados}

O Índice de Validade de Conteúdo [ICV] foi calculado para cada um dos aspectos de adequação da escala ao português acima mencionados, sendo que, para seu cálculo foram estabelecidos os seguintes parâmetros: índices superiores a 0,80 seriam considerados desejáveis; índices entre 0,60 e 0,80, seriam considerados recomendados; índices inferiores a 0,60 seriam considerados aceitos apenas para uso em pesquisa (desaconselhável para uso clínico) (Alexandre \& Coluci, 2011). Sendo assim, qualquer resultado superior a 0,60 pode ser interpretado como indicador de adequação semântica e/ou conceitual dos itens do instrumento, no enquadramento deste estudo. O IVC foi calculado de duas formas: 1. IVC (\% juízes), definido como a "média das proporções dos itens considerados relevantes pelos juízes" (incluindo o instrumento inteiro); 2. Média IVC-i, consiste na média dos valores dos itens calculados separadamente, isto é, soma-se todos os IVC calculados separadamente e divide-se pelo número de itens considerados na avaliação.

\section{Estudo 2 - Investigação da Validade de Estrutura Interna e Confiabilidade da APS-R para o Português Brasileiro}

\section{Participantes}

Utilizou-se uma amostra não probabilística, isto é, uma amostra de conveniência, composta por 787 estudantes universitários brasileiros, com idade média de 25,98 anos $(D P=7,29)$ e com variação de idade entre 17 e 60 anos. Apesar da grande amplitude, $81 \%$ da amostra compreendeu a faixa etária de adultos emergentes (até 29 anos). Os participantes eram majoritariamente do sexo feminino (79,3\%), residentes no estado de Minas Gerais (62\%), São Paulo (15\%), Bahia (8\%), Rio de Janeiro (6\%) e outros $(9 \%)$. Entre os participantes, $76 \%$ estavam matriculados em cursos de graduação em Psicologia, sendo que os $24 \%$ restantes cursavam graduação em diversas áreas do conhecimento, tais como ciências da saúde, exatas e biológicas. O recrutamento dos participantes deu-se por meio do contato prévio com as coordenações dos cursos de graduação, professores e próprios estudantes por metodologia bola de neve. Esse contato foi feito por $e$-mail institucional, telefonemas e apresentação da proposta de pesquisa in loco. Participaram apenas os estudantes que assinaram o Termo de Consentimento Livre e Esclarecido.

\section{Instrumentos}

Almost Perfect Scale-Revised - APS-R (Slaney et al., 2001): instrumento de aplicação coletiva composto por 23 itens que avaliam três dimensões do perfeccionismo. Esses itens devem ser respondidos em uma escala do tipo Likert de 7 pontos que varia do rótulo "discordo totalmente" (1) até "concordo totalmente" (7). A dimensão de Padrões, composta por sete itens, avalia a tendência ao estabelecimento de altos padrões de desempenho e exigência consigo mesmo (ex.: "Espero sempre o melhor de mim”). A dimensão Discrepância é composta por 12 itens que avaliam as diferenças entre os padrões estabelecidos e a percepção que os indivíduos têm de seu desempenho, ou seja, a insatisfação entre o esperado e o alcançado (ex.: "Quando nunca estou satisfeito(a) com o meu desempenho"). A dimensão de Ordem avalia a tendência ao asseio e à organização e é composta por quatro itens. (ex.: "Gosto de sempre ser organizado(a), disciplinado(a)").

Questionário de caracterização sociodemográfica: informações sociais (sexo, idade, escolaridade e outras) e econômicas (renda familiar) dos participantes foram coletados por meio de um questionário elaborado pelos autores do presente estudo.

\section{Procedimentos de Coleta dos Dados}

Os dados foram coletados pelos pesquisadores e por alunos de Psicologia devidamente treinados. As aplicações foram coletivas e ocorreram ao longo ano de 2017 simultaneamente nos estados participantes. As aplicações duraram aproximadamente 40 minutos e foram presenciais. Na etapa da análise de dados, apenas aqueles indivíduos que assinaram o Termo de Consentimento Livre e Esclarecido foram considerados.

\section{Procedimentos de Análise dos Dados}

Primeiramente, foram excluídos os participantes com mais de $5 \%$ de respostas nulas na escala (protocolos excluídos $=26$ ). Esse critério é geralmente adotado para minimizar erros em análises multivariadas (Hair, Black, Babin, Anderson, \& Tatham, 2009; Tabachnick \& Fidell, 2007). Posteriormente à adaptação cultural do instrumento e sua aplicação, a modelagem de equações estruturais [Análise Fatorial Confirmatória (AFC) e Exploratory Structural Equation Modeling (ESEM)] foi utilizada para testar a adequação do modelo estrutural teórico do perfeccionismo trifatorial (Slaney et al., 2001), um modelo alternativo mais parcimonioso (Esforços Perfeccionistas e Preocupações Perfeccionistas) e a proposta bifatorial para a escala. A AFC tem sido usada para confirmação de modelos teóricos acerca de construtos psicológicos. Ao possibilitar verificar o ajuste de modelos teóricos aos dados obtidos essa técnica estatística fornece evidências de validade para medidas (Hair et al., 2009). Para o cálculo de confiabilidade, foi utilizado o ômega de Mcdonald e o alfa de Cronbach, tendo em vista as dimensões da escala representadas no modelo de melhor ajuste.

\section{Modelos Testados:}

1. Os três fatores da escala original correlacionados (Slaney et al., 2001).

2. Modelo alternativo baseado na noção adaptativa/desadaptativa do perfeccionismo (Stoeber, 2018), 
composto por um fator adaptativo que incluiu os itens de Ordem e Padrões e um desadaptativo composto pelos itens de Discrepância. Esse modelo foi baseado na proposta atual de Esforços Perfeccionistas e Preocupações Perfeccionistas, na qual análise da dimensões do perfeccionismo apresentam-se como proxis dessas dimensões teóricas mais gerais que costumam aparecer independente da escala utilizada. A adoção desse modelo para a APS-R significaria a unificação dos escores de Padrões e Ordem.

3. Modelo bifatorial composto por um fator geral explicando a variância dos 23 itens e mais os três fatores originais da escala. Por conter diversas peculiaridades e apresentar-se como novidade para a APS-R, a seguir, é apresentada a justificativa para testagem desse modelo inédito.

\section{Justificativa para Adoção de um Modelo Bifatorial}

As estruturas bifatoriais são representadas por n-dimensões ortogonais propostas pela teoria e por um fator único responsável por explicar a variância não explicada pelas dimensões específicas. É possível obter modelos bifatoriais por meio da modelagem exploratória de equações estruturais (ESEM) (Asparouhov \& Muthen, 2009). Propõe-se que construtos como personalidade e sintomas psicopatológicos, seriam mais bem descritos por esse tipo de análise, pois, a menos que se encontre uma série de sinônimos para representar o traço-alvo os itens desses instrumentos geralmente possuem carga fatorial cruzada. As cargas cruzadas permitem criar parâmetros para avaliar a influência de todas as dimensões sobre os itens. A ESEM vai além da AFE, sendo indicada para uso em fases de criação e confirmação do modelo teórico de escalas e soma-se à AFC como ferramenta de avaliação de modelos teóricos (Booth \& Hughes, 2014). Gäde et al. (2017), com o objetivo de lançar luz às dúvidas acerca da sobreposição dos fatores de segunda ordem encontrados no perfeccionismo (Esforço Perfeccionista e Preocupações Perfeccionistas), investigaram vários modelos estruturais, dentre eles um modelo bifatorial. Embora utilizando uma escala diferente da APS-R, os resultados obtidos apontaram para uma sobreposição entre o fator geral e o de Preocupações Perfeccionistas, desafiando a independência desde fator; enquanto o Esforço Positivo foi capaz de explicar a variância dos itens independentemente do fator geral. Avanços como os citados neste estudo tornam-se possíveis devido ao uso da Modelagem Exploratória com Equações Estruturais (ESEM) para modelos bifatoriais. Assim, é proposta uma estrutura bifatorial para o construto conservando seus três fatores originais. Tal estrutura, caso apresente índices de ajuste adequado, pode corroborar com o uso do escore total da escala já utilizado em alguns estudos.

\section{Parâmetros de Avaliação dos Modelos Testados}

Para a escolha do modelo de melhor aderência aos dados, adotou-se indicação de Hu e Bentler (1999) de que, para além dos índices de ajuste calculados para cada um dos modelos (Figura 1), deve-se optar por aquele que, em comparação com um modelo mais parcimonioso, ainda apresente bons índices de adequação das estatísticas teste, nesse caso, baseadas no qui-quadrado.

Para verificação da adequação dos modelos, foram usados índices de ajustes absolutos, ajuste comparativo e baseados na não centralidade. Os critérios adotados para avaliar e determinar a qualidade dos modelos foram valores da Root Mean Square Error of Approximation (RMSEA) <0,08 (Hair et al., 2009), Tucker Lewis Index (TLI) $>0,90$ (Bentler \& Bonett, 1980) e Comparative Fit Index (CFI)>0,95 (Hu \& Bentler, 1999). Os softwares usados para as análises foram o Mplus 7 (Múthen \& Múthen, 2010) e o JASP versão 0.8.1.2 (JASP Team, 2019).

\section{Resultados}

\section{Etapa 1 - Tradução e Adaptação da APS-R}

Na primeira fase - tradução do inglês para o português, evidenciou-se a necessidade de buscar um equivalente linguístico par ao item 4 ("Neatness is importante to $m e$ "), pois a tradução literal do radical "neat" (asseio) não é de uso comum no Brasil. Na síntese das versões, optou-se pela palavra "limpeza", com a indicação de "asseio" entre parentêses. Os demais itens tiveram poucas diferenças entre as versões traduzidas. Quanto às retrotraduções, houve equivalência de significado referencial e em relação ao instrumento original com concordância variando de $70 \%$ a $100 \%$ entre os tradutores.

Por fim, nas fases três e quatro que dizem respeito à apreciação formal de equivalência e avaliação final por especialistas na área, houve substituição de alguns termos, como advérbios, a fim de evitar repetição excessiva nos itens. Essa medida foi tomada baseando-se nas referências de Borsa, Damásio e Bandeira (2012) para produção e tradução de itens de um instrumento. Pelo fato do idioma do instrumento original se tratar de uma língua em que há representação de um gênero neutro (it), na versão em português foram acrescentadas flexões de gênero para os pronomes (ex.: "(a)”). Para identificação das dimensões da escala, não foi necessário o cálculo Kappa, pois todos os cinco juízes concordaram $(100 \%)$ sobre seu conteúdo e representação. $\mathrm{Na}$ quarta etapa, a versão traduzida mostrou resultados promissores a partir do cálculo do Índice de Validade de Conteúdo. O IVC apresentou índices satisfatórios no tocante a equivalência semântica/idiomática $(0,99) \%)$, cultural $(0,94)$ e conceitual $(0,94)$. O IVC médio apresentou os seguintes valores para equivalência semântica/idiomática $(0,99)$, cultural $(0,94)$ e conceitual $(0,94)$. Todos os índices anteriores foram considerados suficientemente bons. 
Modelo 1 - Tridimensional

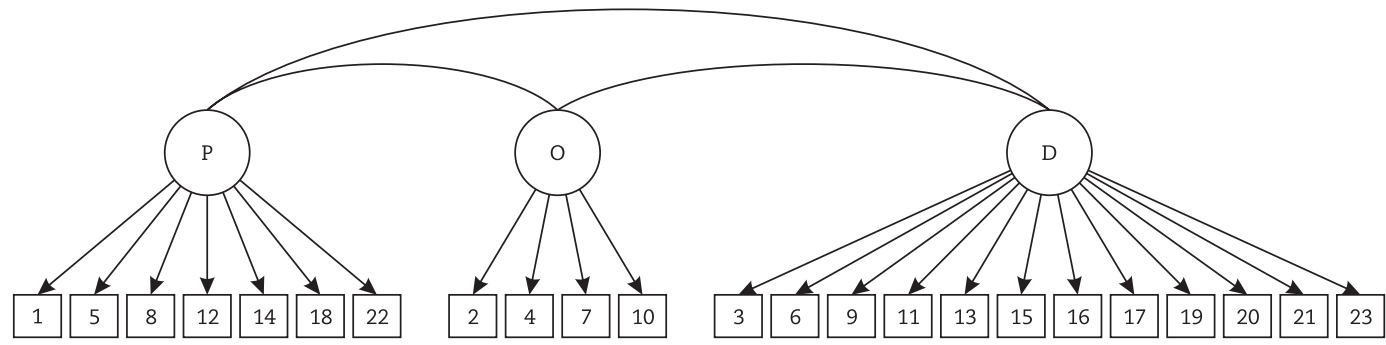

Modelo 2 - Perfeccionismo adaptativo e Desadaptativo

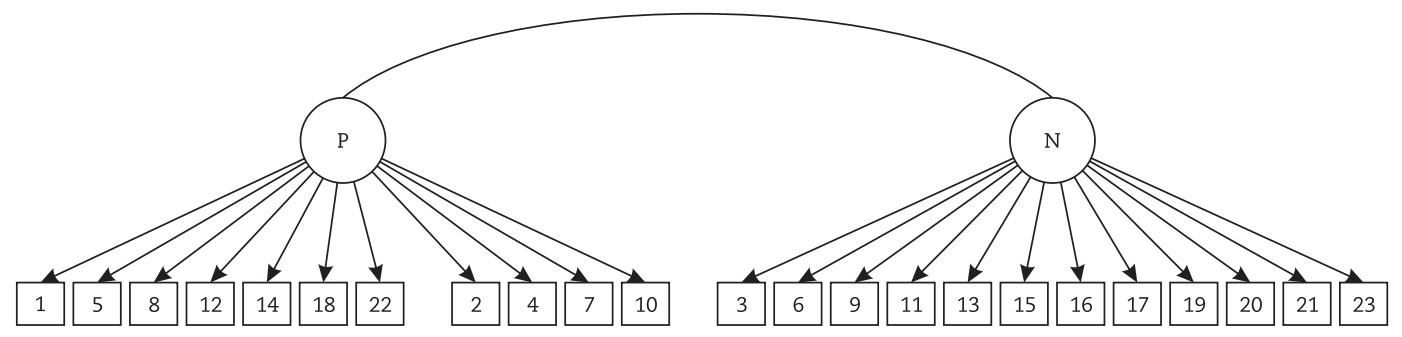

Modelo 3 - Modelo Bifatorial

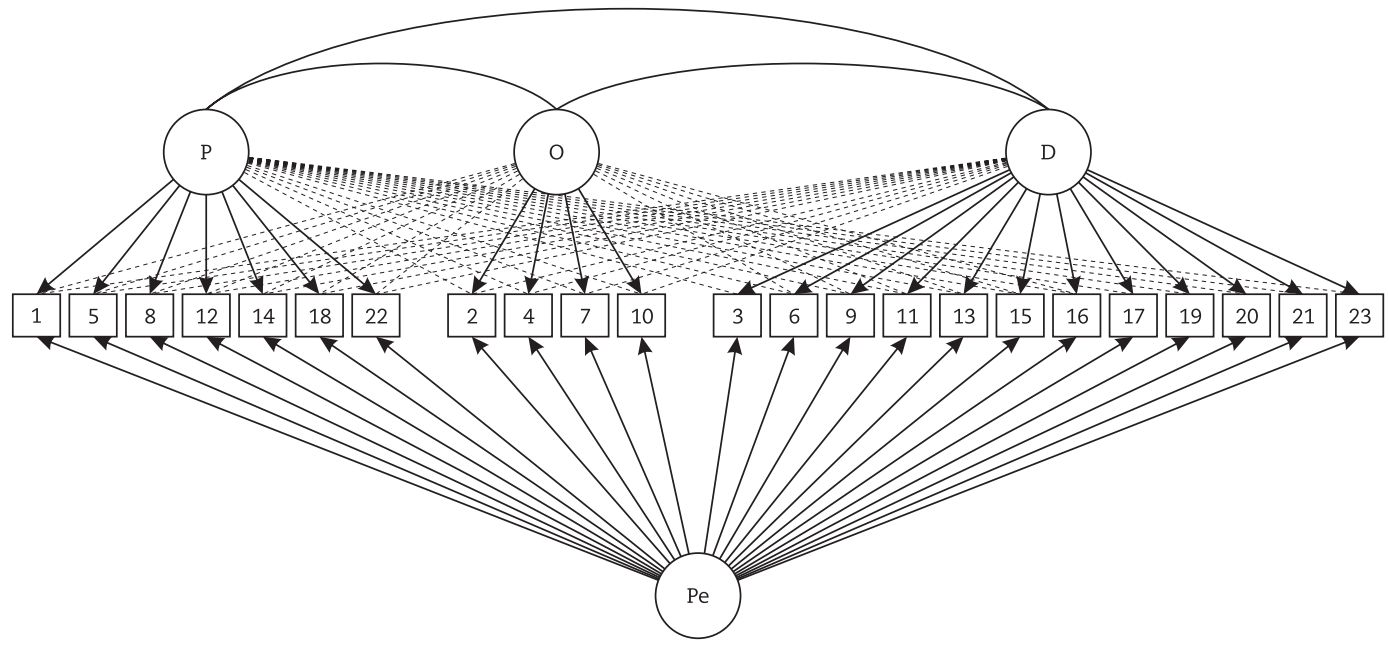

Figura 1. Representação Gráfica dos Modelos Testados $\mathrm{P}=$ Padrões; O=Ordem; D=Discrepância; Pe=Perfeccionismo

\section{Etapa 2 - Evidências de Validade e Confiabilidade da Escala Adaptada Culturalmente}

A fim de se testar a adequação do modelo teórico de três fatores de Slaney et. al (2001) da APS-R versão em português brasileiro, utilizou-se a análise fatorial confirmatória (AFC). O modelo 1 (trifatorial) foi configurado com as dimensões latentes de Padrões, Discrepância e Ordem compostas pelos itens indicados na Figura 1. O Modelo 2 foi composto pela dimensão latente de perfeccionismo adaptativo (itens de Ordem e Padrões) e desadaptativo (itens de discrepância). O Modelo 3 foi semelhante ao Modelo 1, porém configurado para ser bifatorial com uma dimensão geral (Perfeccionismo-Pe) explicando a variância dos itens não explicada pelas dimensões do modelo trifatorial. A técnica utilizada para estimação das cargas foi a da Máxima Verossimilhança Robusta (MLR). Para avaliar a qualidade do ajuste dos modelos propostos, adotou-se como base os índices de $\chi^{2}$, RMSEA, CFI e TLI. Os índices de ajuste de todos os modelos podem ser vistos na Tabela 1. Para o Modelo 1 , os índices foram parcialmente adequados, considerando os padrões mais conservadores (Hu \& Bentler,1999). 
Embora, o CFI e TLI estejam abaixo de 0,90, o RMSEA se apresenta dentro dos limites aceitos levando-se em conta o tamanho da amostra (RMSEA $<0,10$ ) (Hair et al., 2009). A maior parte dos itens apresentou cargas maiores que 0,40 , valor considerado adequado para retenção do item no fator latente em AFC (Hair et al., 2009). Contudo, o item 5 da escala de Padrões demonstrou carga fatorial menor do que 0,40 . O Modelo 2 foi construído para ser mais parcimonioso, contou apenas com duas dimensões e baseou-se nos postulados dos dois tipos de perfeccionismo: adaptativo e desadaptativo perfeccionismo (Egan, Piek, \& Dyck, 2015; Rice et al., 2007). Embora esse modelo tenha sido criado para ser mais parcimonioso, seus índices de ajuste não se mostraram melhores do que Modelo 1 (Tabela 1). O Modelo 3, a proposta bifatorial dos autores do presente estudo, apresentou os melhores índices de ajuste, tendo um bom RMSEA $(<0,05)$ e CFI e TLI maiores do que os demais modelos, indicando sua retenção como melhor modelo. As cargas fatoriais dos itens para o modelo bifatorial podem ser verificadas na Tabela 2. Os itens de discrepância tiveram cargas fatoriais maiores no fator de perfeccionismo geral (todas $>0,40$ ) do que no fator discrepância. Nos critérios BIC e AIC, esse modelo mostrou-se superior aos demais com uma diferença maior que 10 entre os valores. Isso indica que a melhora do modelo estrutural foi significativa.

Os fatores mostraram índices de confiabilidade adequados, tanto no alfa de Cronbach quanto ômega de Mcdonald. A correlação entre fatores variou de pequena à média para os fatores de Padrões e Ordem e Discrepância e foi não significativa entre Discrepância e Ordem (ver Tabela 2).

Tabela 1

Índices de Ajuste dos Modelos

\begin{tabular}{|c|c|c|c|c|c|c|c|c|c|}
\hline Modelo & $\chi^{2}$ & $d f$ & SRMR & AIC & BIC & TLI & CFI & \multicolumn{2}{|c|}{ RMSEA (90\% IC) } \\
\hline Modelo 1- CFA três dimensões & 6571,112 & $253^{*}$ & 0,098 & 63183,3 & 63524 & 0,822 & 0,841 & 0,075 & $(0,071-0,079)$ \\
\hline Modelo 2- CFA duas dime & 6571,11 & $253^{*}$ & 0,108 & 63162,2 & 63587 & 0,763 & 0,787 & 0,087 & $(0,083-0,091)$ \\
\hline Modelo 3- ESEM Bifatorial & 452,371 & $165^{*}$ & 0,025 & 62325,6 & 62951 & 0,944 & 0,964 & 0,047 & $(0,042-0,052)$ \\
\hline
\end{tabular}

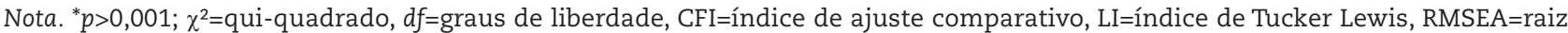
da média dos quadrados dos erros de aproximação. Em negrito o modelo com melhores índices de ajuste

Tabela 2

Modelo Bifatorial da APS-R Brasil - Cargas Fatoriais dos Itens, Confiabilidade das Dimensões e Estatística Descritiva

\begin{tabular}{|c|c|c|c|c|c|c|c|c|}
\hline Itens & $\mathrm{Pg}$ & $\mathrm{P}$ & $\mathrm{D}$ & $\mathrm{O}$ & M & $D P$ & A & $\mathrm{C}$ \\
\hline 1. Mantenho meus padrões ...* & $-0,16$ & 0,51 & 0,15 & 0,16 & 5,14 & 1,42 & $-0,86$ & 0,21 \\
\hline 5. Se você não espera muito de si ... & 0,19 & 0,29 & $-0,00$ & 0,08 & 5,38 & 1,58 & $-1,05$ & 0,45 \\
\hline 8. Tenho expectativas elevadas... & 0,07 & 0,64 & $-0,19$ & 0,05 & 5,73 & 1,40 & $-1,40$ & 1,69 \\
\hline 12. Estabeleço padrões... & 0,49 & 0,54 & $-0,02$ & 0,08 & 4,70 & 1,75 & $-0,46$ & $-0,83$ \\
\hline 14. Espero sempre o melhor... & $-0,02$ & 0,66 & $-0,03$ & 0,12 & 4,97 & 1,77 & $-0,64$ & $-0,63$ \\
\hline 18. Eu tento dar o meu melhor ... & $-0,01$ & 0,48 & 0,15 & 0,26 & 5,73 & 1,41 & $-1,31$ & 1,31 \\
\hline 22. Tenho uma forte necessidade ... & 0,30 & 0,56 & 0,17 & 0,18 & 5,45 & 1,58 & $-0,60$ & $-0,46$ \\
\hline \multicolumn{9}{|l|}{ Discrepância } \\
\hline 3. Frequentemente me sinto frustrado... & 0,50 & 0,04 & $-0,06$ & 0,01 & 5,21 & 1,67 & $-0,80$ & $-0,37$ \\
\hline 6. "O meu melhor" nunca parece... & 0,66 & 0,19 & 0,10 & 0,00 & 4,63 & 1,89 & $-0,40$ & $-1,05$ \\
\hline 9. Poucas vezes correspondo... & 0,69 & $-0,09$ & $-0,42$ & $-0,01$ & 4,25 & 1,69 & $-0,11$ & $-1,00$ \\
\hline 11. Fazer o meu melhor nunca... & 0,77 & 0,12 & 0,09 & 0,05 & 4,38 & 1,95 & $-0,19$ & $-1,22$ \\
\hline 13. Nunca estou satisfeito... & 0,73 & $-0,02$ & 0,27 & $-0,04$ & 3,61 & 1,92 & 0,22 & $-1,19$ \\
\hline 15. Constantemente me preocupo... & 0,52 & 0,26 & $-0,03$ & $-0,03$ & 5,70 & 1,40 & $-1,30$ & 1,36 \\
\hline 16. Meu desempenho raramente ... & 0,76 & $-0,14$ & $-0,19$ & $-0,04$ & 3,92 & 1,79 & 0,13 & $-1,09$ \\
\hline 17. Nunca fico satisfeito... & 0,72 & $-0,01$ & 0,24 & 0,06 & 3,48 & 1,89 & 0,37 & $-1,11$ \\
\hline 19. Raramente sou capaz de atingir... & 0,70 & $-0,14$ & $-0,19$ & $-0,07$ & 3,67 & 1,73 & 0,23 & $-0,98$ \\
\hline 20. Quase nunca estou satisfeito... & 0,85 & $-0,11$ & 0,10 & $-0,05$ & 3,69 & 1,87 & 0,27 & $-1,20$ \\
\hline 21. Quase nunca sinto que o que... & 0,79 & $-0,03$ & 0,14 & $-0,03$ & 3,85 & 1,91 & 0,17 & $-1,27$ \\
\hline 23. Geralmente fico desapontado... & 0,65 & 0,05 & 0,18 & 0,04 & 4,29 & 1,89 & $-0,12$ & $-1,22$ \\
\hline
\end{tabular}


Tabela 2 (continuação)

Modelo Bifatorial da APS-R Brasil - Cargas Fatoriais dos Itens, Confiabilidade das Dimensões e Estatística Descritiva

\begin{tabular}{|c|c|c|c|c|c|c|c|c|c|}
\hline \multicolumn{2}{|l|}{ Itens } & Pg & $\mathrm{P}$ & $\mathrm{D}$ & $\mathrm{O}$ & M & DP & A & $\mathrm{C}$ \\
\hline \multicolumn{10}{|l|}{ Ordem } \\
\hline \multicolumn{2}{|l|}{ 2. Sou uma pessoa... } & $-0,14$ & 0,27 & 0,02 & 0,65 & 4,73 & 1,67 & $-0,58$ & $-0,60$ \\
\hline \multicolumn{2}{|l|}{ 4. Limpeza (asseio) é... } & 0,04 & 0,09 & $-0,01$ & 0,42 & 6,02 & 1,15 & $-1,36$ & 1,80 \\
\hline \multicolumn{2}{|l|}{ 7. Acredito que as coisas... } & 0,13 & 0,14 & 0,00 & 0,57 & 5,42 & 1,37 & $-0,88$ & 0,46 \\
\hline \multicolumn{2}{|l|}{ 10. Gosto de ser sempre... } & 0,07 & 0,28 & 0,03 & 0,80 & 5,07 & 1,54 & $-0,69$ & $-0,30$ \\
\hline \multicolumn{2}{|c|}{ Consistência interna ômega de Mcdonald } & 0,87 & 0,75 & 0,92 & 0,73 & & & & \\
\hline \multicolumn{2}{|l|}{ Consistência Interna alfa de Cronbach } & 0,86 & 0,74 & 0,91 & 0,71 & & & & \\
\hline \multirow[t]{3}{*}{ Correlações entre as dimensões ${ }^{* *}$} & Padrões & & - & 0,21 & 0,52 & & & & \\
\hline & Discrepância & & & - & 0,01 & & & & \\
\hline & Ordem & & & & - & & & & \\
\hline
\end{tabular}

Nota. $\mathrm{Pg}=$ perfeccionismo geral; $\mathrm{P}=$ padrões; $\mathrm{D}=$ discrepância; $\mathrm{O}=$ Ordem; $\mathrm{M}=$ média; $\mathrm{DP}=\mathrm{Desvio}$ Padrão; $\mathrm{A}=$ assimetria; $\mathrm{C}=\mathrm{Curtose}$. Em negrito as cargas fatoriais nas dimensões originalmente propostas.

* Para ter acesso à escala completa, entre em contato com os autores pelos endereços eletrônicos: marmansura@gmail.com ou cbneufeld@usp.br

** Calculado com o modelo original em AFC, pois o modelo bifatorial não permite a correlação das dimensões latentes

\section{Discussão}

O objetivo do estudo foi traduzir, adaptar culturalmente e verificar as propriedades psicométricas da Almost Perfect Scale - Revised para uma amostra de brasileiros. Esse objetivo foi dividido em duas etapas, a primeira delas para verificar a validade de conteúdo da versão brasileira da escala e a segunda para buscar evidências de validade psicométrica, por meio da análise de estrutura interna e confiabilidade. Esse segundo processo se deu por meio de análise fatorial confirmatória e cálculo de confiabilidade. A escala traduzida apresentou equivalência semântica, cultural e idiomática quando comparada à versão original, conforme indicaram os índices de validade de conteúdo.

A qualidade empírica dos itens da escala foi satisfatória, com exceção do item 5 - ("Se você não espera muito de si mesmo, nunca terá sucesso") que não pareceu representar a dimensão de Padrões tão bem quanto os demais itens dessa dimensão (carga fatorial $=0,30$ ). A respeito disso, embora esse item tenha apresentado cargas adequadas em outros estudos em ambas AFE e AFC (ex.: Cazan, 2016; Park, 2009; Slaney et al., 2001) em outras adaptações da escala para línguas românicas, o item 5 também não apresentou carga adequada ou manteve cargas ambíguas nos fatores Padrões e Ordem ocasionando sua exclusão em algumas versões da APS-R (Arana et al., 2009; Sastre-Riba et al., 2016). Em uma amostra específica (adolescentes de 13 a 19 anos), esse item mostrou-se inadequado mesmo para falantes do inglês (Vandiver \& Worrell, 2002). No presente estudo, uma explicação para baixa qualidade do item, apesar de sua aprovação na etapa de avaliação dos juízes, é que, do ponto de vista semântico, sua compreensão tenha sido dificultada pela dupla negativa contida na sentença. As duplas negativas são sentenças mais complexas e incomuns no português, assim recomenda-se evitar traduções literais como essa e alterar o item para que seu sentido original seja preservado e de fácil entendimento para população-alvo (Borsa et al., 2012). Contudo, embora o item de número 5 apresente cargas fatoriais abaixo do recomendado (5. Se você não espera muito de si...) a simulação de sua exclusão não aumentou os valores dos índices de confiabilidade (alfa de Cronbach $=0,74$ e ômega de McDonald =0,75) e não causou melhora nos índices de ajuste dos modelos testados. Assim, a indicação é que o item não seja retirado, mas modificado, dado que seu conteúdo semântico e conceitual correspondeu ao esperado. Ademais, a busca incessante por alto desempenho expressa por esse item é uma característica central no perfeccionismo (Slaney et al., 2001). Dito isso, o item seria melhor expresso por: "Você só obtém sucesso se esperar/exigir muito de si mesmo". Estudos complementares deverão ser conduzidos a fim de verificar a adequação da nova versão do item.

A escala adaptada apresentou bons índices de confiabilidade para todas as suas dimensões. Destaca-se que os 23 itens, quando analisados em conjunto, exibiram alta consistência interna $(\alpha=0,87$ e $\omega=0,87)$, o que poderia apontar para uma dimensão geral latente, capaz de explicar as congruências entre os itens. Adicionalmente, as dimensões de Padrões e Discrepância possuem correlações positivas $(r=0,21)$, mais uma evidência a respeito de um fator geral.

Quanto ao ajuste do melhor modelo para os dados brasileiros (AFC e ESEM), foram investigadas as soluções estruturais mais recorrentes na literatura, tanto no que se refere aos estudos psicométricos da escala 
quanto em discussões conceituais sobre o perfeccionismo (Stoeber, 2018). Os parâmetros utilizados se basearam no modelo original da escala contra o modelo com dois fatores e o modelo bifatorial (ver Figura 1). Os resultados de estudos psicométricos das versões adaptadas da APS-R têm demonstrado índices de ajuste do modelo trifatorial limítrofes, levando em consideração padrões mais conservadores para avaliação de modelos, como RMSEA $\geq 0,05$ e CFI e TLI acima de 0,95 (Hair et al., 2009; Hu \& Bentler, 1999). Por exemplo, Cazan (2016) relatou RMSEA de 0,07 e CFI de 0,87 para o modelo de duas dimensões da escala. Em uma amostra indiana, os modelos de três dimensões também apresentaram valores limítrofes $\chi^{2}(223,132)=311,84, p<0,001, \mathrm{CFI}=0,93$, $\mathrm{SRMR}=0,90$, RMSEA $=0,04$ (Wang et al., 2012). Disso resultou que a estrutura bifatorial foi mais apropriada para representação dos dados, mesmo que o modelo original da escala tenha sido replicado em certa medida. Por outro lado, em amostras estado-unidenses, a estrutura latente da escala se mostrou-se invariável, sempre apontando para uma estrutura com três fatores (Mobley et al., 2005; Slaney et al., 2001). Isso levanta a questão sobre as influências da cultura nas manifestações do perfeccionismo. Sabe-se, por exemplo, que diferentes estilos e modelos parentais têm influência sobre o perfeccionismo, sendo esse um fator que varia bastante culturalmente (Walton, Hibbard, Coughlin, \& Coyl-Shepherd, 2018)

As adaptações da escala e sua variação estrutural em diferentes estudos torna mais difícil a comparação entre diferentes países. No estudo atual, por exemplo, a APS-R pareceu capturar mais aspectos negativos do perfeccionismo do que positivos, o que pode ser observado ao se analisar a contribuição do fator geral denominado "perfeccionismo", para os itens de discrepância. O fator geral explicou em maior proporção a variância dos itens de discrepância do que a própria dimensão ortogonal às demais. Os itens 6 ("O meu melhor nunca parece ser o bastante para mim.") e 11 ("Fazer o meu melhor nunca parece ser o suficiente.") apresentaram carga negativas em Discrepância e o item 15 ("Constantemente me preocupo em não corresponder às minhas próprias expectativas.") mostrou carga maior em Padrões. Esse resultado indica que a tendência geral medida pela escala se relaciona às preocupações perfeccionistas, ou seja, a tendência em experimentar ansiedade e disforia frente às imposições de altos padrões de desempenho. Em termo clínicos, essa configuração deve-se à presença frequente tanto de esforços perfeccionistas quanto de preocupações perfeccionistas, no mesmo indivíduo, o que Stoeber (2018) denomina como "tipo misto de perfeccionista".

Quanto ao uso da escala adotando-se um modelo bifatorial, embora a proposta para a APS-R Brasil seja nova, a ideia por trás não é uma novidade, sendo que esse tipo de estrutura tem sido testado para diversos construtos psicológicos. Por exemplo, Smith et al. (2017) analisaram um modelo bifatorial do perfeccionismo usando da versão americana da APS-R e as dimensões das escalas de Frost e Hewitt (Frost et al., 1990; Hewitt, Flett, Turnbull-Donovan, \& Mikail, 1991). Além de encontrarem melhores índices ajuste para tal modelo, os autores indicaram que a maior parte da variância comum sobre as dimensões principais do perfeccionismo foi atribuída à um fator latente geral. Embora esse resultado indique a possibilidade de uso de um escore único das escalas de perfeccionismo, os dados mostrados aqui sugerem cautela, pois, os itens das dimensões de Padrões e, principalmente, Ordem apresentaram cargas baixas no fator geral do modelo. Quanto ao fator Ordem, é possível discutir sua participação como dimensão estruturante do conceito de perfeccionismo (Frost et al., 1990; Mobley et al., 2005; Stoeber et al., 2006).

Embora o modelo original da escala seja moderadamente bom, ele apresenta limitações quando comparado ao modelo bifatorial. Este permite verificar a independência de cada uma das dimensões do perfeccionismo sob desfechos importantes, controlando-se o peso do fator geral, além de permitir justificar o uso de um escore geral para determinar a severidade do perfeccionismo. Não obstante, o modelo original não permite a investigação de aspectos gerais e específicos do perfeccionismo ao mesmo tempo. A literatura recentemente tem indicado a necessidade do uso de um modelo bifatorial antes de se fazer inferências sobre os esforços perfeccionistas e as preocupações perfeccionistas, pois o fator geral, ortogonal aos fatores específicos podem explicar os padrões de correlação de esforços perfeccionistas com desfechos negativos e a falta de correlação de preocupações perfeccionistas com desfechos também negativos em alguns estudos (Smith \& Saklofsk, 2017). Esses padrões contrariam as proposições de adaptativo e desadaptativo propostas para o perfeccionismo, questionando se de fato altos padrões e esforço são características inócuas desse construto. Nesse caso, a adoção do modelo bifatorial contribui não somente para a clínica (perfeccionismo clínico), mas também para pesquisas sobre o construto e seus componentes e desfechos. Não obstante, desde a criação das escalas de perfeccionismo, escores gerais das escalas de perfeccionismo já eram usados em alguns estudos sem uma justificativa estrutural (ex.: Frost et al., 1990).

Desse modo, na amostra do presente estudo, o perfeccionismo pareceu se manifestar, como indica a estrutura bifatorial, mais representado pela discrepância do que por seus considerados positivos aspectos positivos - padrões e ordem. Dada a complexidade do construto e a configuração das cargas fatoriais, assim como a própria definição do perfeccionismo (ex.: altos padrões e alto criticismo), o uso de um fator geral é indicado a fim de controlar e separar os efeitos que cada uma das dimensões têm sobre as outras. Adicionalmente, as dimensões demonstraram não ter funcionamento independente. Assim, a correlação geralmente encontrada entre os aspectos mais positivos e mais negativos do perfeccionismo 
podem ser explicadas por esse fator geral (Gäde et al., 2017; Smith et al., 2017).

Estudos de validade de critério tanto com amostras clínicas, quanto com amostras gerais e mais heterogêneas poderiam contribuir para uma melhor compreensão dos aspectos positivos e negativos do perfeccionismo e da sua estruturação como construto. É possível, que, por meio da comparação de grupos critérios (como indivíduos com diagnóstico de transtornos da ansiedade ou obsessivo-compulsivo com um grupo controle, sem psicopatologias), seja possível hipotetizar se a Discrepância é a única dimensão fundamentalmente negativa do perfeccionismo. Ademais, outros estudos poderiam associar o fator geral do perfeccionismo e os fatores específicos (Padrões e Ordem) a medidas de desfecho positivas e negativas, visando compreender melhor a rede nomológica do construto. Hoje, é possível classificar indivíduos como não perfeccionistas, perfeccionistas adaptativos e perfeccionistas desadaptativos usando as dimensões da APS-R como independentes (Rice \& Ashby, 2007). O modelo bifatorial permite que essa classificação seja feita a partir da criação de um ponto de corte para o fator geral da APS-R. Isso torna essa categorização mais próxima do que seria considerado perfeccionismo clínico, destacando sua característica transdiagnóstica para além das especificidades de cada uma das dimensões da escala (Egan et al., 2014). Adicionalmente, modelos bifatoriais das psicopatologias e características desadaptativas têm auxiliados clínicos e pesquisadores a explicar comorbidades e sobreposição entre psicopatologias (Constantinou et al., 2019).

Em resumo, apesar de o estudo atual ter testado um modelo novo e pouco usual quando se trata da escala de Slaney et al. (2001) e ter sido o primeiro a apresentar uma versão adaptada de uma escala objetiva de perfeccionismo para o Brasil, ainda restam dúvidas quanto a melhor maneira de representar o construto na população brasileira. A despeito dos modelos confirmatórios, a escala apresentou evidências de validade estrutural com cargas fatoriais adequadas para os itens e valores adequados de consistências interna. Ademais, o presente estudo trouxe evidências suportando o uso clínico da escala com um escore total "Perfeccionismo Clínico" e escores separados para cada uma das outras dimensões (Egan et al., 2014).

Quando às limitações, a pesquisa atual representa apenas uma região geopolítica brasileira e teve uma amostra majoritariamente feminina. Além disso, o estudo do perfeccionismo no Brasil está apenas se iniciando e as suposições acerca do modelo teórico mais adequado não pode ser generalizado nem interpretado sem a devida cautela. Estudos que incluam mais de um instrumento de avaliação do perfeccionismo nas mesmas amostras poderão lançar luz sobre o melhor modelo estrutural para o construto, conforme aponta Stoeber (2018).

Apesar disso, a APS-R pode ser considerada um instrumento clínico importante, pois, pode ser utilizada no rastreio de níveis de elevados perfeccionismo, para caracterização de aspectos negativos mais presentes em pacientes e como acompanhamento de mudanças nos níveis de perfeccionismo desses indivíduos no curso da psicoterapia. Essas utilizações se destacam principalmente porque as medidas de perfeccionismo têm mostrado eficientes no acompanhamento e predição de desfechos clínicos, como depressão, índice de tentativas de suicídio e responsividade ao tratamento psicoterápico (Törnblom, Werbart, \& Rydelius, 2013).

No sentido de sanar as dúvidas sobre o melhor modelo teórico do perfeccionismo se faz necessário estudar sua rede nomológica. Portanto, a indicação é que as próximas pesquisas utilizem outras escalas em conjunto com a APS-R Brasil e tenham foco no construto Perfeccionismo e seu valor preditivo para desfechos como psicopatologias, bem-estar e saúde geral.

\section{Considerações Finais}

O perfeccionismo é um construto complexo, assim como sua rede de relações com psicopatologias e diferenças individuais. Apesar de sua importância para contextos clínicos e de saúde mental, nenhuma escala que permitisse sua avaliação objetiva havia ainda sido adaptada para o Brasil até a presente data. O estudo atual é o primeiro de uma série a publicar dados sobre o perfeccionismo em amostras brasileiras. Os resultados alcançados e o modelo bifatorial proposto estão em concordância com as concepções adaptativas e desadaptativas do perfeccionismo, ou seja, o modelo de perfeccionismo $2 \times 2$ - esforços perfeccionistas combinados com preocupações perfeccionistas - proposto por Gaudreau e Thompson (2010) e revisto por Stoeber (2018) e que poderá servir de base para estudos futuros acerca do construto no país.

\section{Agradecimentos}

Não há menções.

\section{Financiamento}

A presente pesquisa não recebeu nenhuma fonte de financiamento sendo custeada com recursos dos próprios autores.

\section{Contribuições dos autores}

Todos os autores contribuíram substancialmente para a elaboração do delineamento da pesquisa, análise e interpretação dos dados, bem como, para a revisão textual e aprovação da versão final deste estudo. Todos os autores assumem responsabilidade pública pelo conteúdo do manuscrito

\section{Disponibilidade dos dados e materiais}

Todos os dados e sintaxes gerados e analisados durante esta pesquisa serão tratados com total sigilo 
devido às exigências do Comitê de Ética em Pesquisa em Seres Humanos. O conjunto de dados e sintaxes que apoiam as conclusões deste artigo não estão disponíveis no momento. Contudo, se houver interesse pelos dados, a solicitação poderá ser feita para o autor de correspondência principal e será avaliada pelos autores.

\section{Conflito de interesses}

Os autores declaram que não há conflitos de interesses.

\section{Referências}

Alexandre, N. M. C., \& Coluci, M. Z. O. (2011). Validade de conteúdo nos processos de construção e adaptação de instrumentos de medidas. Ciência e Saúde Coletiva, 16(7), 3061-3068. doi: 10.1590/S1413-81232011000800006

Arana, F. G., Keegan, E., \& Rutsztein, G. (2009). Adaptación de una medida multidimensional de perfeccionismo: La Almost Perfect ScaleRevised (APS-R). Un estudio preliminar sobre sus propiedades psicométricas en una muestra de estudiantes universitarios argentinos. Revista Evaluar, 9(1), 35-53. doi: 10.35670/1667-4545.v9.n1.463

Asparouhov, T., \& Muthén, B. (2009). Exploratory structural equation modeling. Structural Equation Modeling: a Multidisciplinary Journal, 16(3), 397-438. doi: 10.1080/10705510903008204

Bentler, P. M., \& Bonett, D. G. (1980). Significance tests and goodness of fit in the analysis of covariance structures. Psychological Bulletin, 88(3), 588-606. doi:10.1037/0033-2909.88.3.588

Booth, T., \& Hughes, D. J. (2014). Exploratory Structural Equation Modeling of Personality Data. Assessment, 21(3), 260-271. doi: $10.1177 / 1073191114528029$

Borsa, J. C., Damásio, B. F., \& Bandeira, D. R. (2012). Adaptação e validação de instrumentos psicológicos entre culturas: Algumas considerações. Paideia, 22(53), 423-432. doi: 10.1590/1982-43272253201314

Constantinou, M. P., Goodyer, I. M., Eisler, I., Butler, S., Kraam, A., Scott, S., ... Fonagy, P. (2019). Changes in General and Specific Psychopathology Factors Over a Psychosocial Intervention. Journal of the American Academy of Child and Adolescent Psychiatry, 58(8), 776786. doi: 10.1016/j.jaac.2018.11.011

Cazan, A. (2016). Almost Perfect Scale - Validity of a Perfectionism Scale on a Romanian University Sample. Romanian Journal of Experimental Applied Psychology, 7(1), 367-371. Disponível em: http://www.revista.experimentala.ro/files/vol7si1p2015/PSIWORLD_2015_ PROCEEDINGS_RJEAP ART a79.pdf

Chan, D. W. (2010). Perfectionism among Chinese gifted and nongifted students in Hong Kong: The Use of the Revised Almost Perfect Scale. Journal for the Education of the Gifted. Orange, 34(1), 68-98. doi: 10.1177/016235321003400104

Egan, S. J., Piek, J. P., \& Dyck, M. J. (2015). Positive and negative perfectionism and the big five personality factors. Behaviour Change, 32(02), 104-113. doi: 10.1017/bec.2015.3

Egan, S. J., Wade, T. D., Shafran, R., \& Antony, M. M. (2014). Cognitive-behavioral treatment of perfectionism. Cognitive-behavioral treatment of perfectionism. New York: Guilford Publications

Flett, G. L., \& Hewitt, P. L. (2016). Still measuring perfectionism after all these years: Reflections and an introduction to the special issue on advances in the assessment of perfectionism. Journal of Psychoeducational Assessment, 34(7), 615-619. doi: 10.1177/0734282916651540

Frost, R. O., Marten, P., Lahart, C., \& Rosenblate, R. (1990). The dimensions of perfectionism. Cognitive Therapy and Research, 14(5), 449468. doi: 10.1007/BF01172967

Gäde, J. C., Schermelleh-Engel, K., \& Klein, A. G. (2017). Disentangling the common variance of perfectionistic strivings and perfectionistic concerns: A bifactor model of perfectionism. Frontiers in Psychology, 8(FEB), 1-13. doi: 10.3389/fpsyg.2017.00160

Gaudreau, P., \& Thompson, A. (2010). Testing a $2 \times 2$ model of dispositional perfectionism. Personality and Individual Differences, 48(5), 532537. doi: 10.1016/j.paid.2009.11.031

Hair, J. F., Black, W. C., Babin, B. J., Anderson, R. E., \& Tatham, R. L. (2009). Análise multivariada de dados. Porto Alegre: Bookman.

Hewitt, P. L., Flett, G. L., Turnbull-Donovan, W., \& Mikail, S. F. (1991). The Multidimensional Perfectionism Scale: Reliability, validity, and psychometric properties in psychiatric samples. Psychological Assessment, 3(3), 464-468. doi: 10.1037/1040-3590.3.3.464

Hu, L. T., \& Bentler, P. M. (1999). Cutoff criteria for fit indexes in covariance structure analysis: Conventional criteria versus new alternatives. Structural Equation Modeling, 6(1), 1-55. doi: 10.1080/10705519909540118

International Test Commission (ITC). (2016). The International Test Commission Guidelines on the Security of Tests, Examinations, and Other Assessments: International Test Commission (ITC). International Journal of Testing, 16(3), 181-204. doi: 10.1080/15305058.2015.1111221

JASP team. (2019). JASP (version 0.10. 2) [computer software]. See https://jasp-stats. org.

Mobley, M., Slaney, R. B., \& Rice, K. G. (2005). Cultural validity of the Almost Perfect Scale - Revised for African American college students. Journal of Counseling Psychology, 52(4), 629-639. doi: 10.1037/0022-0167.52.4.629

Newby, J., Pitura, V. A., Penney, A. M., Klein, R. G., Flett, G. L., \& Hewitt, P. L. (2017). Neuroticism and perfectionism as predictors of social anxiety. Personality and Individual Differences, 106, 263-267. doi: 10.1016/j.paid.2016.10.057

Park, H. J. (2009). Validation of the Almost Perfect Scale - Revised. The Korean Journal of Counseling and Psychotherapy, 21(1), 131-149. Recuperado de http://www.krcpa.or.kr/data/rental/board_upload/thesis/21_1PDF/08.pdf

Pinto, A., Dargani, N., Wheaton, M. G., Cervoni, C., Rees, C. S., \& Egan, S. J. (2017). Perfectionism in obsessive-compulsive disorder and related disorders: What should treating clinicians know? Journal of Obsessive-Compulsive and Related Disorders, 12, 102-108. doi: 10.1016/j.jocrd.2017.01.001

Rice, K. G., \& Ashby, J. S. (2007). An efficient method for classifying perfectionists. Journal of Counseling Psychology, 54(1), 72-85. doi: $10.1037 / 0022-0167.54 .1 .72$ 
Rice, K. G., Ashby, J. S., \& Slaney, R. B. (2007). Perfectionism and the Five-Factor Model of Personality. Assessment, 14(4), 385-398. doi: 10.1177/1073191107303217

Sastre-Riba, S., Pérez-Albéniz, A., \& Fonseca-Pedrero, E. (2016). Assessing perfectionism in children and adolescents: Psychometric properties of the Almost Perfect Scale Revised. Learning and Individual Differences, 49, 386-392. doi: 10.1016/j.lindif.2016.06.022

Sherry, S. B., Richards, J. E., Sherry, D. L., \& Stewart, S. H. (2014). Self-critical perfectionism is a vulnerability factor for depression but not anxiety: A 12-month, 3-wave longitudinal study. Journal of Research in Personality, 52, 1-5, doi: 10.1016/j.jrp.2014.05.004

Slaney, R. B., Mobley, M., Trippi, J., Ashby. J.. \& Johnson, D. G. (1996). The Almost Perfect Scale - Revised. Unpublished manuscript. The Pennsylvania State University, University Park. PA

Slaney, R., Rice, K., Mobley, M., Trippi, J., \& Ashby, J. S. (2001). The Revised Almost Perfect Scale. Measurement and Evaluation in Counseling E Development, 34(3), 130-145.

Smith, M. M., \& Saklofske, D. H. (2017). The structure of multidimensional perfectionism: Support for a Bifactor Model With a Dominant General Factor The Structure of Multidimensional Perfectionism. Support for a Bifactor Model With a Dominant General Factor, Journal of Personality Assessment, 99(3), 297-303. doi: 10.1080/00223891.2016.1208209

Smith, M. M., Saklofske, D. H., Yan, G., \& Sherry, S. B. (2017). Does Perfectionism Predict Depression, Anxiety, Stress, and Life Satisfaction After Controlling for Neuroticism? Journal of Individual Differences, 38, 63-70. doi: 10.1027/1614-0001/a000223

Stairs, A. M., Smith, G. T., Zapolski, T. C. B., Combs, J. L., \& Settles, R. E. (2012). Clarifying the construct of perfectionism. Assessment, 19(2), 146-66. doi: 10.1177/1073191111411663

Stoeber, J. (2018). The psychology of perfectionism: Critical issues, open questions, and future directions. Em J. Stoeber (Org.), The psychology of perfectionism: Theory, research, applications (pp. 333-352). London: Routledge.

Stoeber, J., Otto, K., Bardi, A., Giner-Sorolla, R., Hamilton-West, K., Joor-Mann, J., ... Weick, M. (2006). Positive conceptions of perfectionism: Approaches, evidence, challenges. Personality and Social Psychology Review, 10(4), 295-319. doi: 10.1207/s15327957pspr1004

Törnblom, A. W., Werbart, A., \& Rydelius, P. A. (2013). Shame behind the masks: The parents' perspective on their sons' suicide. Archives of Suicide Research, 17(3), 242-261. doi: 10.1080/13811118.2013.805644

Vandiver, B. J., \& Worrell, F. C. (2002). The reliability and validity of scores on the Almost Perfect Scale - Revised with academically talented middle school students. The Journal of Secondary Gifted Education, 13(3), 108-119. doi: 10.4219/jsge-2002-372

Walton, G. E., Hibbard, D. R., Coughlin, C., \& Coyl-Shepherd, D. D. (2018). Parenting, personality, and culture as predictors of perfectionism. Current Psychology, 1-13. doi: 10.1007\%2Fs12144-018-9793-y

Wang, K. T., Puri, R., Slaney, R. B., Methikalam, B., \& Chadha, N. (2012). Cultural Validity of Perfectionism Among Indian Students. Measurement and Evaluation in Counseling and Development, 45(1), 32-48. doi: 10.1177/0748175611423109

\section{Sobre os autores}

Flávio Henrique dos Reis Soares é psicólogo, mestre em psicologia do desenvolvimento e doutorando no Programa de PósGraduação em Psicologia: Cognição e Comportamento, Universidade Federal de Minas Gerais, Brasil.

Anelisa Vaz de Carvalho é doutoranda do Programa de Pós-Graduação em Psicologia da Faculdade de Filosofia, Ciências e Letras de Ribeirão Preto, Universidade do Estado de São Paulo, Campus Ribeirão Preto.

Eduardo Keegan é psicólogo e doutor em Psicologia na Universidade de Buenos Aires (UBA). Atualmente é Diretor de Carreira da Especialização em Psicologia Clínica e Terapia Cognitivo-Comportamental da Faculdade de Psicologia da Universidade de Buenos Aires.

Carmem Beatriz Neufeld é psicóloga, Professora do Departamento de Psicologia, Programa de Pós-Graduação em Psicologia da Faculdade de Filosofia, Ciências e Letras de Ribeirão Preto, Universidade do Estado de São Paulo, Campus Ribeirão Preto.

Marcela Mansur-Alves é psicóloga, Professora do Departamento de Psicologia, Programa de Pós-Graduação em Psicologia: Cognição e Comportamento, Faculdade de Filosofia e Ciências Humanas, Universidade Federal de Minas Gerais.

\section{Como citar este artigo}

Soares et al. (2020). Adaptação e Validação da Escala de Perfeccionismo Almost Perfect Scale - Revised para o Português Brasileiro. Avaliação Psicológica, 19(3), 310-321. http://dx.doi.org/10.15689/ap.2020.1903.17282.09 